\title{
Models for highway traffic and their connections to thermodynamics.
}

\author{
Hans Weber ${ }^{1}$, Reinhard Mahnke ${ }^{2}$, Julia Hinkel ${ }^{3}$, and Anders Strömberg ${ }^{4}$ \\ 1 Luleå University of Technology, Department of Physics, SE-97187 Luleå, Sweden \\ Hans. Weber@ltu.se \\ 2 Rostock University, Institute of Physics, D - 18051 Rostock, Germany \\ reinhard.mahnke@uni-rostock.de \\ 3 Rostock University, Institute of Physics, D - 18051 Rostock, Germany \\ julia.hinkel@uni-rostock.de \\ 4 Luleå University of Technology, Department of Physics, SE-97187 Luleå, Sweden \\ anesot-0@student.luth.se
}

\begin{abstract}
Models for highway traffic are studied by numerical simulations. Of special interest is the spontaneous formation of traffic jams. In a thermodynamic system the traffic jam would correspond to the dense phase (liquid) and the free flowing traffic would correspond to the gas phase. Both phases depending on the density of cars can be present at the same time. A model for a single lane circular road has been studied. The model is called the optimal velocity model (OVM) and was developed by Bando, Sugiyama, et al. We propose here is a reformulation of the OVM into a description in terms of potential energy functions forming a kind of Hamiltonian for the system. This will however not be globally defined Hamiltonian but a locally defined one as it is a dynamical model. The model defined by this Hamiltonian will be suitable for MonteCarlo simulations.
\end{abstract}

\section{Bando Model}

We report a suggested reformulation of the Bando Model [1, 2], to a model including a kind of thermodynamics. The Bando model is a deterministic model for traffic flow. We restrict the work to a 1 dimensional single lane circular road shown in figure 1.

Velocity of car $i$ is denoted by $v_{i}$ and position by $x_{i}$, (in a dimensionless formulation the set of equations to the right the velocity is $u$ and position is $y$ ). The Bando model is defined by the following set of equations 


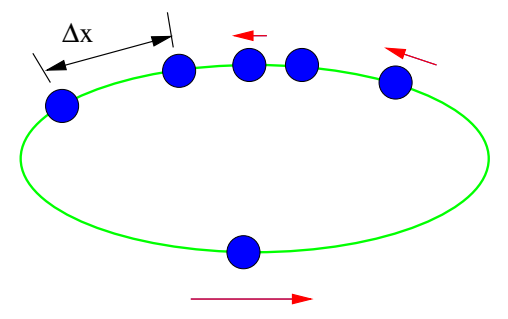

Fig. 1. One dimensional circular road with periodic boundary conditions. The cars are represented by blue filled circles, their velocity's are marked by red arrows and the headaway distance is marked by $\Delta x$.

$$
\begin{cases}\frac{d}{d t} v_{i}=\frac{1}{\tau}\left(v_{o p t}\left(\Delta x_{i}\right)-v_{i}\right) & \frac{d}{d t} u_{i}=\left(u_{o p t}\left(\Delta y_{i}\right)-y_{i}\right) \\ \frac{d}{d t} x_{i}=v_{i} & \frac{d}{d t} y_{i}=\frac{1}{b} u_{i} \\ v_{\text {opt }}\left(\Delta x_{i}\right)=v_{\max } \frac{(\Delta x)^{2}}{D^{2}+(\Delta x)^{2}} & u_{\text {opt }}\left(\Delta y_{i}\right)=\frac{(\Delta y)^{2}}{1+(\Delta y)^{2}} \\ & b=\frac{D}{v_{\max }}\end{cases}
$$

The control parameters are the maximal velocity $v_{\max }$, the time scale $\tau$ and the interaction distance $D$. The optimal velocity $v_{o p t}(\Delta x)$ is a function of headway (bumper-to-bumper) distance $\Delta x_{i}=x_{i+1}-x_{i}$. The average density of cars is $c=\frac{N}{L}$.

The acceleration of the cars is given by $a_{i}=\frac{d v}{d t}$ and is given by the Bando model (eq 1). This acceleration corresponds to a force on the car according to Newton's second law $F=m a=\frac{d v}{d t}$. Finally we get the potential energy $V_{p o t}$ associated with the $F$ as we know the displacement it acts over.

This potential energy can be used to reformulate the Bando model into a Hamiltonian fot the system of cars. It should be noted however that the Hamiltonian obtained is not a true Hamiltonian as it is only locally defined. But all the least we can still use it to perform Monte-Carlo simulations.

Below we show some earlier numerical results for the Bando model [3], where the equations where integrated out with 4th order Runge-Kutta method. Under certain conditions the traffic separates into two phases. A dense (=jam) and a dilute (=free flow) one. Very much like a liquid-gas transition, use the difference in densities as order parameter. The results in figure 2 show a coexisting dense and dilute phase for certain values of $b$ and $c$, where the cars move in either a jam $u_{\text {min }}$ or in a free flow $u_{\max }$. In corresponding phase diagram can be seen in figure 3 .

\section{Monte Carlo Simulations}

With Monte Carlo simulations one can analyze many complex (many degrees of freedom) problems such as: magnetic systems, gases, super conductors, 


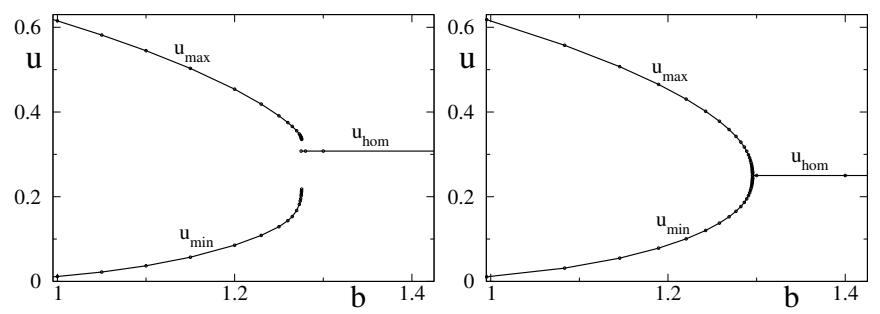

Fig. 2. The figure to the left shows a sub critical bifurcation diagram $(c=1.5)$ and figure to the right shows a critical bifurcation diagram $(c=\sqrt{3})(N=60)$.

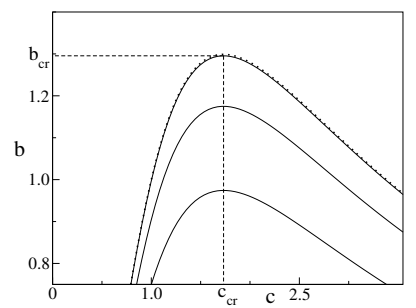

Fig. 3. Phase diagram of the Bando Model. The critical value for $b$ is $b_{c r}=3 \sqrt{3} / 4$.

atoms, nuclear decay, telephone switchboards,... We will take an example the 2D Ising model. This is a model for magnetic systems and also other cases with two states of the configurations variables like a binary alloy. In figure 2 a grafical representation of the 2 dimensional Ising model on a square lattice is shown.

The Ising model in 2 dimensions has an exact solution, the famous Onsager solution [4] and in principle one should not need to perform Monte-Carlo simulations on it. But it has become very famous and it has reached a position within condensed matter physics similar to the Bohr atomic model for hydrogen.

Every state of the Ising model has an energy according to the Hamiltonian of the system

$$
H=-\sum_{<i, j>} S_{i} \cdot S_{j} .
$$

The thermodynamic properties are given by the partition function $Z=$ $\sum_{l} e^{-H_{l} / k_{B} T}$. From $Z$ we can calculate "any" thermodynamic property of the system. Most (nearly all) systems are however to complicated to be solved analytically in a closed form and we have to revert to Monte - Carlo simulations (see [5]) in order to learn more about the model in question.

Now we will describe how to do Monte-Carlo simulations in practice. For a configuration of spins $S_{i}$, the Metropolis procedure is: 


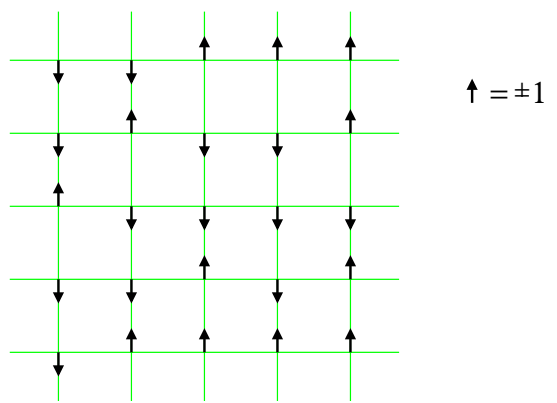

Fig. 4. The 2 dimensional Ising model on a square lattice. At each lattice site there is a configuration variable $S_{i j}= \pm 1$ shown here as arrows.

1. Generate a new state by a change to one of the spins $S_{j} \rightarrow S_{j}+\Delta S_{j}$. For the Ising model

2. Calculate the energy difference $\Delta E$.

3. Accept the new state if $\Delta E<0$, else if $\Delta E>0$, accept the new state if $r<e^{-\Delta E / k_{B} T}$ where $r$ is a random number $r \in[0,1]$, otherwise keep the old value.

4. Goto step 1.

Monte-Carlo usually used for equilibrium properties, but can be used for dynamics as well. There are other Monte - Carlo procedures as well as the heat bath method.

\section{Driven system, an Example}

As it is not directly clear that one should be able to get any sensible results from Monte-Carlo simulations for a driven system. A driven system is not in equilibrium and thereby we are no assured that equilibrium methods should apply. But we will show an example of how it can be done. One of the authors has performed a Monte-Carlo simulation of a current-voltage (IV) characteristics for a superconducting film. Just some short remarks about the system In a SC film vortex pairs a thermally excited. Vortices interact logarithmically $V(r)=\ln (r)$ and hence system is a 2D Coulomb gas. A Monte-Carlo move consists of adding \pm- pairs (charge neutral) at random position and random orientation.

Dynamics: IV current - voltage characteristics. The electric field $\rightarrow$ Lorentz force gives created pairs different energy according to their orientation.

The energy contribution due to the Lorentz force introduces a local part into the Hamiltonian $\rightarrow$ No global Hamiltonian!

$$
V \propto I^{a} \quad a=3 \text { at } T_{c}
$$



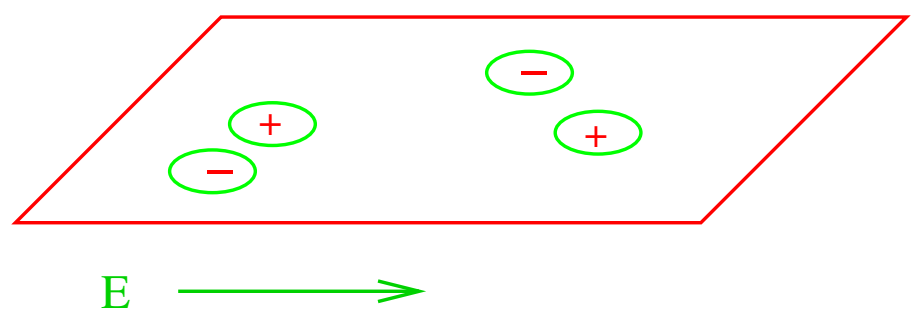

Fig. 5. A two dimensional superconductor with 2 vortex pairs in it. The electrical field is in the direction of $E$.
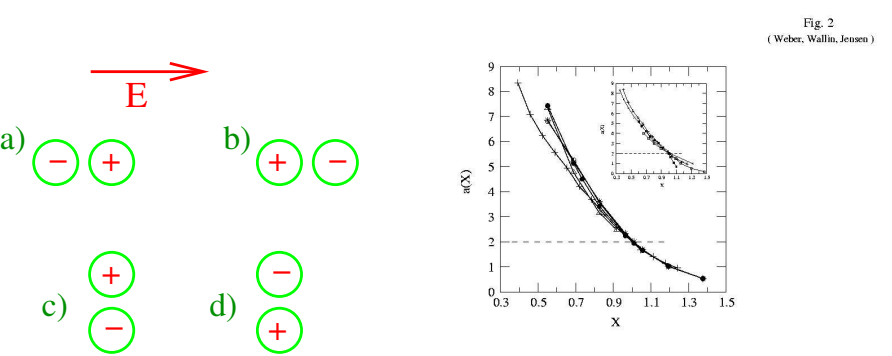

Fig. 6. To the left four possible orientations of a vortex pair are seen with respect to the driving electrical field $E$. The energy of a particular pair depends on its orientation. Configurations a) and b) have different energy and c) and d) are not effected by the presence of the field $E$. To the right Monte-Carlo simulation results are shown together with experimental results, the different curves fall on top of each other suggesting that we can recover the experimentall facts with a simple driven model. $X$ is the reduced temperature $\frac{T}{T_{c}}$. Figure to the right is from [6].

Non linear $I V$ characteristics from experiments and Monte-Carlo simulations compare very well.

\section{Traffic flow}

Now to the Metropolis procedure for the cars. The basic idea is simple we reformulate the Bando model in terms of locally defined potential energys.

1. For a car $i$ make a random change in velocity $\Delta v \in\left[-\Delta v_{\max }, \Delta v_{\max }\right]$.

2. The force $F$ is known from the Bando model (eq. 1) to the car ahead and behind.

3. Calculate the change in energy $\Delta E$ (pot + kinetic) due to the proposed change in velocity $\Delta v$ of the car.

4. Use Metropolis to determine if the change $\Delta v$ is accepted.

5. Move the car with either its new or old velocity in time step $\Delta t$. 
There is an extra parameter in the problem the ratio $m / T$ where $m$ is the mass of the car and $T$ is the temperature. However note, $T$ is not real temperature it is a meassure of the strength of fluctuations. The potential energy $V_{\text {pot }}$ is also unusual as it is a function of both velocity $v_{i}$ and position $x_{i}$.

We have made test runs with a preliminary program for the Bando model defined in terms of potential and kinetic energys. These preliminary runs have been restricted to only take the potential energy into account, as this corresponds closly to the Bando model. In the figure 7 we show the potential energy of a single car in a system consisting of only 3 cars. These three cars are
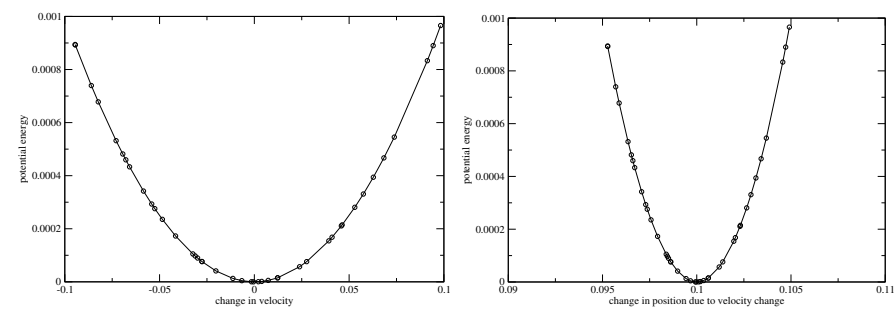

Fig. 7. The potential energy for a single car in a system with $N=3$ and $L=30$. The left figure shows the potential energy of a car at fixed position as a function of its velocity. The right figure shows the potential energy of a car with fixed velocity as a function of position.

set to the homogeneous solution $v$ (equidistant) $=1.000$ and $c=\Delta x=10.0$. In figure 7 the functional form of the potential is that of a parabola with its minimum at the homogeneous solution.

\section{References}

1. M. Bando, K. Hasebe, A. Nakayama, A. Shibata, Y. Sugiyama: Japan J. Indust. and Appl. Math. 11, 203, 1994; Phys. Rev. E 51, 1035, 1995.

2. M. Bando, K. Hasebe, K. Nakanishi, A. Nakayama, A. Shibata, Y. Sugiyama: J. Phys. I France 5, 1389, 1995.

3. Jevgenijs Kaupužs, Hans Weber, Aliaksei Konash and Reinhard Mahnke, Applications to Traffic Breakdown on Highways, ECMI (Riga 2002). In: Progress in Industrial Mathematics at ECMI 2002 (Eds.: A. Buikis, R. Ciegis, A. D. Fitt), pp. 133-138, Springer-Verlag, Berlin, 2004 (ISDN 3-540-40113-X).

4. Onsager, L., Phys. Rev. 65, 117-149, 1944.

5. M. E. J. Newman \& G. T. Barkema: Monte Carlo Methods in Statistical Physics, (Clarendon Press, Oxford 1999) pp 45-85.

6. Hans Weber, Mats Wallin and Henrik Jeldtoft Jensen, Phys. Rev. B 53, 8566, 1996. 